\title{
Existence of Solutions for Fractional Differential Inclusions with Antiperiodic Boundary Conditions
}

\author{
Bashir Ahmad $^{1}$ and Victoria Otero-Espinar ${ }^{2}$ \\ ${ }^{1}$ Department of Mathematics, Faculty of Science, King Abdulaziz University, P.O. Box 80203, \\ Jeddah 21589, Saudi Arabia \\ ${ }^{2}$ Departamento de Análisis Matemático, Facultad de Matemáticas, Universidad de Santiago de Compostela, \\ 15782 Santiago de Compostela, Spain
}

Correspondence should be addressed to Victoria Otero-Espinar, mvictoria.otero@usc.es

Received 21 January 2009; Revised 6 March 2009; Accepted 18 March 2009

Recommended by Juan J. Nieto

We study the existence of solutions for a class of fractional differential inclusions with anti-periodic boundary conditions. The main result of the paper is based on Bohnenblust-Karlins fixed point theorem. Some applications of the main result are also discussed.

Copyright (C) 2009 B. Ahmad and V. Otero-Espinar. This is an open access article distributed under the Creative Commons Attribution License, which permits unrestricted use, distribution, and reproduction in any medium, provided the original work is properly cited.

\section{Introduction}

In some cases and real world problems, fractional-order models are found to be more adequate than integer-order models as fractional derivatives provide an excellent tool for the description of memory and hereditary properties of various materials and processes. The mathematical modelling of systems and processes in the fields of physics, chemistry, aerodynamics, electro dynamics of complex medium, polymer rheology, and so forth, involves derivatives of fractional order. In consequence, the subject of fractional differential equations is gaining much importance and attention. For details and examples, see [1-14] and the references therein.

Antiperiodic boundary value problems have recently received considerable attention as antiperiodic boundary conditions appear in numerous situations, for instance, see [15-22].

Differential inclusions arise in the mathematical modelling of certain problems in economics, optimal control, and so forth. and are widely studied by many authors, see [2327] and the references therein. For some recent development on differential inclusions, we refer the reader to the references [28-32]. 
Chang and Nieto [33] discussed the existence of solutions for the fractional boundary value problem:

$$
\begin{gathered}
{ }_{0}^{c} D_{t}^{\delta} y(t) \in F(t, y(t)), \quad t \in[0,1], \delta \in(1,2), \\
y(0)=\alpha, \quad y(1)=\beta, \quad \alpha, \beta \neq 0 .
\end{gathered}
$$

In this paper, we consider the following fractional differential inclusions with antiperiodic boundary conditions

$$
\begin{gathered}
{ }^{c} D^{q} x(t) \in F(t, x(t)), \quad t \in[0, T], T>0,1<q \leq 2, \\
x(0)=-x(T), \quad x^{\prime}(0)=-x^{\prime}(T),
\end{gathered}
$$

where ${ }^{c} D^{q}$ denotes the Caputo fractional derivative of order $q, F:[0, T] \times \mathbb{R} \rightarrow 2^{\mathbb{R}} \backslash\{\varnothing\}$. Bohnenblust-Karlin fixed point theorem is applied to prove the existence of solutions of (1.2).

\section{Preliminaries}

Let $C([0, T])$ denote a Banach space of continuous functions from $[0, T]$ into $\mathbb{R}$ with the norm $\|x\|=\sup _{t \in[0, T]}\{|x(t)|\}$. Let $L^{1}([0, T], \mathbb{R})$ be the Banach space of functions $x:[0, T] \rightarrow \mathbb{R}$ which are Lebesgue integrable and normed by $\|x\|_{L_{1}}=\int_{0}^{T}|x(t)| d t$.

Now we recall some basic definitions on multivalued maps [34, 35].

Let $(X,\|\cdot\|)$ be a Banach space. Then a multivalued map $G: X \rightarrow 2^{X}$ is convex (closed) valued if $G(x)$ is convex (closed) for all $x \in X$. The map $G$ is bounded on bounded sets if $G(\mathbb{B})=\bigcup_{x \in \mathbb{B}} G(x)$ is bounded in $X$ for any bounded set $\mathbb{B}$ of $X$ (i.e., $\sup _{x \in \mathbb{B}}\{\sup \{|y|: y \in$ $G(x)\}\}<\infty$ ). $G$ is called upper semicontinuous (u.s.c.) on $X$ if for each $x_{0} \in X$, the set $G\left(x_{0}\right)$ is a nonempty closed subset of $X$, and if for each open set $\mathbb{B}$ of $X$ containing $G\left(x_{0}\right)$, there exists an open neighborhood $\mathcal{N}$ of $x_{0}$ such that $G(\mathcal{N} \subseteq \mathbb{B}$. $G$ is said to be completely continuous if $G(\mathbb{B})$ is relatively compact for every bounded subset $\mathbb{B}$ of $X$. If the multivalued map $G$ is completely continuous with nonempty compact values, then $G$ is u.s.c. if and only if $G$ has a closed graph, that is, $x_{n} \rightarrow x_{*}, y_{n} \rightarrow y_{*}, y_{n} \in G\left(x_{n}\right)$ imply $y_{*} \in G\left(x_{*}\right)$. In the following study, $B C C(X)$ denotes the set of all nonempty bounded, closed, and convex subset of $X$. $G$ has a fixed point if there is $x \in X$ such that $x \in G(x)$.

Let us record some definitions on fractional calculus $[8,11,13]$.

Definition 2.1. For a function $g:[0, \infty) \rightarrow \mathbb{R}$, the Caputo derivative of fractional order $q>0$ is defined as

$$
{ }^{c} D^{q} g(t)=\frac{1}{\Gamma(n-q)} \int_{0}^{t}(t-s)^{n-q-1} g^{(n)}(s) d s, \quad n-1<q<n, n=[q]+1
$$

where $[q]$ denotes the integer part of the real number $q$ and $\Gamma$ denotes the gamma function. 
Definition 2.2. The Riemann-Liouville fractional integral of order $q>0$ for a function $g$ is defined as

$$
I^{q} g(t)=\frac{1}{\Gamma(q)} \int_{0}^{t} \frac{g(s)}{(t-s)^{1-q}} d s, \quad q>0
$$

provided the right-hand side is pointwise defined on $(0, \infty)$.

Definition 2.3. The Riemann-Liouville fractional derivative of order $q>0$ for a function $g$ is defined by

$$
D^{q} g(t)=\frac{1}{\Gamma(n-q)}\left(\frac{d}{d t}\right)^{n} \int_{0}^{t} \frac{g(s)}{(t-s)^{q-n+1}} d s, \quad n=[q]+1
$$

provided the right-hand side is pointwise defined on $(0, \infty)$.

In passing, we remark that the Caputo derivative becomes the conventional $n$th derivative of the function as $q \rightarrow n$ and the initial conditions for fractional differential equations retain the same form as that of ordinary differential equations with integer derivatives. On the other hand, the Riemann-Liouville fractional derivative could hardly produce the physical interpretation of the initial conditions required for the initial value problems involving fractional differential equations (the same applies to the boundary value problems of fractional differential equations). Moreover, the Caputo derivative for a constant is zero while the Riemann-Liouville fractional derivative of a constant is nonzero. For more details, see [13].

For the forthcoming analysis, we need the following assumptions:

$\left(\mathrm{A}_{1}\right)$ let $F:[0, T] \times \mathbb{R} \rightarrow B C C(\mathbb{R}) ;(t, x) \rightarrow f(t, x)$ be measurable with respect to $t$ for each $x \in \mathbb{R}$, u.s.c. with respect to $x$ for a.e. $t \in[0, T]$, and for each fixed $x \in \mathbb{R}$, the set $S_{F, y}:=\left\{f \in L^{1}([0, T], \mathbb{R}): f(t) \in F(t, x)\right.$ for a.e. $\left.t \in[0, T]\right\}$ is nonempty,

$\left(\mathrm{A}_{2}\right)$ for each $r>0$, there exists a function $m_{r} \in L^{1}\left([0, T], \mathbb{R}_{+}\right)$such that $\|F(t, x)\|=$ $\sup \{|v|: v(t) \in F(t, x)\} \leq m_{r}(t)$ for each $(t, x) \in[0, T] \times \mathbb{R}$ with $|x| \leq r$, and

$$
\liminf _{r \rightarrow+\infty}\left(\frac{\int_{0}^{T} m_{r}(t) d t}{r}\right)=r<\infty
$$

where $m_{r}$ depends on $r$. For example, for $F(t, x)=x$, we have $m_{r}(t)=r$ and hence $\gamma=T$. If $F(t, x)=x^{2}$, then $\gamma$ is not finite.

Definition $2.4([16,33])$. A function $x \in C([0, T])$ is a solution of the problem (1.2) if there exists a function $f \in L^{1}([0, T], \mathbb{R})$ such that $f(t) \in F(t, x(t))$ a.e. on $[0, T]$ and

$$
\begin{aligned}
x(t)= & \int_{0}^{t} \frac{(t-s)^{q-1}}{\Gamma(q)} f(s) d s-\frac{1}{2} \int_{0}^{T} \frac{(T-s)^{q-1}}{\Gamma(q)} f(s) d s \\
& +\frac{1}{4}(T-2 t) \int_{0}^{T} \frac{(T-s)^{q-2}}{\Gamma(q-1)} f(s) d s,
\end{aligned}
$$


which, in terms of Green's function $G(t, s)$, can be expressed as

$$
x(t)=\int_{0}^{T} G(t, s) f(s) d s
$$

where

$$
G(t, s)= \begin{cases}-\frac{(T-s)^{q-1}}{2 \Gamma(q)}+\frac{(T-2 t)(T-s)^{q-2}}{4 \Gamma(q-1)}, & 0<t<s<T, \\ \frac{(t-s)^{q-1}-\frac{1}{2}(T-s)^{q-1}}{\Gamma(q)}+\frac{(T-2 t)(T-s)^{q-2}}{4 \Gamma(q-1)}, & 0<s<t<T .\end{cases}
$$

Here, we remark that the Green's function $G(t, s)$ for $q=2$ takes the form (see [22])

$$
G(t, s)= \begin{cases}\frac{1}{4}(-T-2 t+2 s), & 0<t<s<T \\ \frac{1}{4}(-T+2 t-2 s), & 0<s<t<T\end{cases}
$$

Now we state the following lemmas which are necessary to establish the main result of the paper.

Lemma 2.5 (Bohnenblust-Karlin [36]). Let D be a nonempty subset of a Banach space $X$, which is bounded, closed, and convex. Suppose that $G: D \rightarrow 2^{X} \backslash\{0\}$ is u.s.c. with closed, convex values such that $G(D) \subset D$ and $\overline{G(D)}$ is compact. Then $G$ has a fixed point.

Lemma 2.6 ([37]). Let I be a compact real interval. Let $F$ be a multivalued map satisfying $\left(A_{1}\right)$ and let $\Theta$ be linear continuous from $L^{1}(I, \mathbb{R}) \rightarrow C(I)$, then the operator $\Theta \circ S_{F}: C(I) \rightarrow$ $B C C(C(I)), x \mapsto\left(\Theta \circ S_{F}\right)(x)=\Theta\left(S_{F, x}\right)$ is a closed graph operator in $C(I) \times C(I)$.

\section{Main Result}

Theorem 3.1. Suppose that the assumptions $\left(A_{1}\right)$ and $\left(A_{2}\right)$ are satisfied, and

$$
r<\frac{4 \Gamma(q)}{(5+q) T^{q-1}}
$$

Then the antiperiodic problem (1.2) has at least one solution on $[0, T]$. 
Proof. To transform the problem (1.2) into a fixed point problem, we define a multivalued $\operatorname{map} \Omega: C([0, T]) \rightarrow 2^{C([0, T])}$ as

$$
\begin{aligned}
\Omega(x)=\left\{h \in C([0, T]): h(t)=\int_{0}^{t} \frac{(t-s)^{q-1}}{\Gamma(q)} f(s) d s-\frac{1}{2} \int_{0}^{T} \frac{(T-s)^{q-1}}{\Gamma(q)} f(s) d s\right. \\
\left.+\frac{1}{4}(T-2 t) \int_{0}^{T} \frac{(T-s)^{q-2}}{\Gamma(q-1)} f(s) d s, f \in S_{F, x}\right\} .
\end{aligned}
$$

Now we prove that $\Omega$ satisfies all the assumptions of Lemma 2.6, and thus $\Omega$ has a fixed point which is a solution of the problem (1.2). As a first step, we show that $\Omega(x)$ is convex for each $x \in C([0, T])$. For that, let $h_{1}, h_{2} \in \Omega(x)$. Then there exist $f_{1}, f_{2} \in S_{F, x}$ such that for each $t \in[0, T]$, we have

$$
\begin{aligned}
h_{i}(t)= & \int_{0}^{t} \frac{(t-s)^{q-1}}{\Gamma(q)} f_{i}(s) d s-\frac{1}{2} \int_{0}^{T} \frac{(T-s)^{q-1}}{\Gamma(q)} f_{i}(s) d s \\
& +\frac{1}{4}(T-2 t) \int_{0}^{T} \frac{(T-s)^{q-2}}{\Gamma(q-1)} f_{i}(s) d s, \quad i=1,2 .
\end{aligned}
$$

Let $0 \leq \lambda \leq 1$. Then, for each $t \in J$, we have

$$
\begin{aligned}
{\left[\lambda h_{1}+(1-\lambda) h_{2}\right](t)=} & \int_{0}^{t} \frac{(t-s)^{q-1}}{\Gamma(q)}\left[\lambda f_{1}(s)+(1-\lambda) f_{2}(s)\right] d s \\
& -\frac{1}{2} \int_{0}^{T} \frac{(T-s)^{q-1}}{\Gamma(q)}\left[\lambda f_{1}(s)+(1-\lambda) f_{2}(s)\right] d s \\
& +\frac{1}{4}(T-2 t) \int_{0}^{T} \frac{(T-s)^{q-2}}{\Gamma(q-1)}\left[\lambda f_{1}(s)+(1-\lambda) f_{2}(s)\right] d s .
\end{aligned}
$$

Since $S_{F, x}$ is convex ( $F$ has convex values), therefore it follows that $\lambda h_{1}+(1-\lambda) h_{2} \in \Omega(x)$.

In order to show that $\Omega(x)$ is closed for each $x \in C([0, T])$, let $\left\{u_{n}\right\}_{n \geq 0} \in \Omega(x)$ be such that $u_{n} \rightarrow u(n \rightarrow \infty)$ in $C([0, T])$. Then $u \in C([0, T])$ and there exists a $v_{n} \in S_{F, x}$ such that

$$
\begin{aligned}
u_{n}(t)= & \int_{0}^{t} \frac{(t-s)^{q-1}}{\Gamma(q)} v_{n}(s) d s-\frac{1}{2} \int_{0}^{T} \frac{(T-s)^{q-1}}{\Gamma(q)} v_{n}(s) d s \\
& +\frac{1}{4}(T-2 t) \int_{0}^{T} \frac{(T-s)^{q-2}}{\Gamma(q-1)} v_{n}(s) d s .
\end{aligned}
$$


As $F$ has compact values, we pass onto a subsequence to obtain that $v_{n}$ converges to $v$ in $L^{1}\left([0, T], \mathbb{R}_{+}\right)$. Thus, $v \in S_{F, x}$ and

$$
\begin{aligned}
u_{n}(t) \longrightarrow u(t)= & \int_{0}^{t} \frac{(t-s)^{q-1}}{\Gamma(q)} v(s) d s-\frac{1}{2} \int_{0}^{T} \frac{(T-s)^{q-1}}{\Gamma(q)} v(s) d s \\
& +\frac{1}{4}(T-2 t) \int_{0}^{T} \frac{(T-s)^{q-2}}{\Gamma(q-1)} v(s) d s .
\end{aligned}
$$

Hence $u \in \Omega(x)$.

Next we show that there exists a positive number $r$ such that $\Omega\left(B_{r}\right) \subseteq B_{r}$, where $B_{r}=$ $\{x \in C([0, T]):\|x\| \leq r\}$. Clearly $B_{r}$ is a bounded closed convex set in $C([0, T])$ for each positive constant $r$. If it is not true, then for each positive number $r$, there exists a function $x_{r} \in B_{r}, h_{r} \in \Omega\left(x_{r}\right)$ with $\left\|\Omega\left(x_{r}\right)\right\|>r$, and

$$
\begin{aligned}
h_{r}(t)= & \int_{0}^{t} \frac{(t-s)^{q-1}}{\Gamma(q)} f_{r}(s) d s-\frac{1}{2} \int_{0}^{T} \frac{(T-s)^{q-1}}{\Gamma(q)} f_{r}(s) d s \\
& +\frac{1}{4}(T-2 t) \int_{0}^{T} \frac{(T-s)^{q-2}}{\Gamma(q-1)} f_{r}(s) d s, \quad \text { for some } f_{r} \in S_{F, x_{r}} .
\end{aligned}
$$

On the other hand, in view of $\left(A_{2}\right)$, we have

$$
\begin{aligned}
r< & \left\|\Omega\left(x_{r}\right)\right\| \\
\leq & \int_{0}^{t} \frac{|t-s|^{q-1}}{\Gamma(q)}\left|f_{r}(s)\right| d s+\frac{1}{2} \int_{0}^{T} \frac{|T-s|^{q-1}}{\Gamma(q)}\left|f_{r}(s)\right| d s \\
& +\frac{1}{4}|T-2 t| \int_{0}^{T} \frac{|T-s|^{q-2}}{\Gamma(q-1)}\left|f_{r}(s)\right| d s \\
\leq & \frac{T^{q-1}}{\Gamma(q)} \int_{0}^{T} m_{r}(s) d s+\frac{T^{q-1}}{2 \Gamma(q)} \int_{0}^{T} m_{r}(s) d s+\frac{T^{q-1}}{4 \Gamma(q-1)} \int_{0}^{T} m_{r}(s) d s \\
= & \frac{T^{q-1}(5+q)}{4 \Gamma(q)} \int_{0}^{T} m_{r}(s) d s,
\end{aligned}
$$

where we have used the fact that

$$
\begin{aligned}
& \left|(T-2 t)(T-s)^{q-2}\right| \leq\left|(T-t)(T-s)^{q-2}\right| \leq|(T-t)|^{q-1}, \quad \text { for } t<s, \\
& \left|(T-2 t)(T-s)^{q-2}\right| \leq\left|(T-t)(T-s)^{q-2}\right| \leq|(T-s)|^{q-1}, \quad \text { for } t \geq s .
\end{aligned}
$$

Dividing both sides of (3.8) by $r$ and taking the lower limit as $r \rightarrow \infty$, we find that $r \geq$ $4 \Gamma(q) /(5+q) T^{q-1}$, which contradicts (3.1). Hence there exists a positive number $r^{\prime}$ such that $\Omega\left(B r^{\prime}\right) \subseteq B r^{\prime}$. 
Now we show that $\Omega\left(B_{r^{\prime}}\right)$ is equicontinuous. Let $t^{\prime}, t^{\prime \prime} \in[0, T]$ with $t^{\prime}<t^{\prime \prime}$. Let $x \in B_{r^{\prime}}$ and $h \in \Omega(x)$, then there exists $f \in S_{F, x}$ such that for each $t \in[0, T]$, we have

$$
h(t)=\int_{0}^{t} \frac{(t-s)^{q-1}}{\Gamma(q)} f(s) d s-\frac{1}{2} \int_{0}^{T} \frac{(T-s)^{q-1}}{\Gamma(q)} f(s) d s+\frac{1}{4}(T-2 t) \int_{0}^{T} \frac{(T-s)^{q-2}}{\Gamma(q-1)} f(s) d s .
$$

Using (3.8), we obtain

$$
\begin{aligned}
\left|h\left(t^{\prime \prime}\right)-h\left(t^{\prime}\right)\right|= & \mid \int_{0}^{t^{\prime \prime}} \frac{\left(t^{\prime \prime}-s\right)^{q-1}}{\Gamma(q)} f(s) d s+\frac{1}{4}\left(T-2 t^{\prime \prime}\right) \int_{0}^{T} \frac{(T-s)^{q-2}}{\Gamma(q-1)} f(s) d s \\
& -\int_{0}^{t^{\prime}} \frac{\left(t^{\prime}-s\right)^{q-1}}{\Gamma(q)} f(s) d s-\frac{1}{4}\left(T-2 t^{\prime}\right) \int_{0}^{T} \frac{(T-s)^{q-2}}{\Gamma(q-1)} f(s) d s \mid \\
\leq & \left|\int_{0}^{t^{\prime}} \frac{\left.\left(t^{\prime \prime}-s\right)^{q-1}-\left(t^{\prime}-s\right)^{q-1}\right]}{\Gamma(q)} f(s) d s\right|+\left|\int_{t^{\prime}}^{t^{\prime \prime}} \frac{\left(t^{\prime \prime}-s\right)^{q-1}}{\Gamma(q)} f(s) d s\right| \\
& +\left|-\frac{\left(t^{\prime \prime}-t^{\prime}\right)}{2} \int_{0}^{T} \frac{(T-s)^{q-2}}{\Gamma(q-1)} f(s) d s\right| \\
\leq & \frac{1}{\Gamma(q)} \int_{0}^{t^{\prime}}\left|\left(t^{\prime \prime}-s\right)^{q-1}-\left(t^{\prime}-s\right)^{q-1}\right| m_{r^{\prime}}(s) d s+\frac{T^{q-1}}{\Gamma(q)} \int_{t^{\prime}}^{t^{\prime \prime}} m_{r^{\prime}}(s) d s \\
& +\frac{\left(t^{\prime \prime}-t^{\prime}\right) T^{q-2}}{2 \Gamma(q-1)} \int_{0}^{T} m_{r^{\prime}}(s) d s .
\end{aligned}
$$

Obviously the right-hand side of the above inequality tends to zero independently of $x \in B_{r^{\prime}}$ as $t^{\prime \prime} \rightarrow t^{\prime}$. Thus, $\Omega$ is equicontinuous.

As $\Omega$ satisfies the above assumptions, therefore it follows by Ascoli-Arzela theorem that $\Omega$ is a compact multivalued map.

Finally, we show that $\Omega$ has a closed graph. Let $x_{n} \rightarrow x_{*}, h_{n} \in \Omega\left(x_{n}\right)$ and $h_{n} \rightarrow h_{*}$. We will show that $h_{*} \in \Omega\left(x_{*}\right)$. By the relation $h_{n} \in \Omega\left(x_{n}\right)$, we mean that there exists $f_{n} \in S_{F, x_{n}}$ such that for each $t \in[0, T]$,

$$
\begin{aligned}
h_{n}(t)= & \int_{0}^{t} \frac{(t-s)^{q-1}}{\Gamma(q)} f_{n}(s) d s-\frac{1}{2} \int_{0}^{T} \frac{(T-s)^{q-1}}{\Gamma(q)} f_{n}(s) d s \\
& +\frac{1}{4}(T-2 t) \int_{0}^{T} \frac{(T-s)^{q-2}}{\Gamma(q-1)} f_{n}(s) d s .
\end{aligned}
$$


Thus we need to show that there exists $f_{*} \in S_{F, x_{*}}$ such that for each $t \in[0, T]$,

$$
\begin{aligned}
h_{*}(t)= & \int_{0}^{t} \frac{(t-s)^{q-1}}{\Gamma(q)} f_{*}(s) d s-\frac{1}{2} \int_{0}^{T} \frac{(T-s)^{q-1}}{\Gamma(q)} f_{*}(s) d s \\
& +\frac{1}{4}(T-2 t) \int_{0}^{T} \frac{(T-s)^{q-2}}{\Gamma(q-1)} f_{*}(s) d s .
\end{aligned}
$$

Let us consider the continuous linear operator $\Theta: L^{1}([0, T], \mathbb{R}) \rightarrow C([0, T])$ so that

$$
\begin{aligned}
f \longmapsto \Theta(f)(t)= & \int_{0}^{t} \frac{(t-s)^{q-1}}{\Gamma(q)} f(s) d s-\frac{1}{2} \int_{0}^{T} \frac{(T-s)^{q-1}}{\Gamma(q)} f(s) d s \\
& +\frac{1}{4}(T-2 t) \int_{0}^{T} \frac{(T-s)^{q-2}}{\Gamma(q-1)} f(s) d s .
\end{aligned}
$$

Observe that

$$
\begin{aligned}
\left\|h_{n}(t)-h_{*}(t)\right\|=\| & \int_{0}^{t} \frac{(t-s)^{q-1}}{\Gamma(q)}\left(f_{n}(s)-f_{*}(s)\right) d s-\frac{1}{2} \int_{0}^{T} \frac{(T-s)^{q-1}}{\Gamma(q)}\left(f_{n}(s)-f_{*}(s)\right) d s \\
& +\frac{1}{4}(T-2 t) \int_{0}^{T} \frac{(T-s)^{q-2}}{\Gamma(q-1)}\left(f_{n}(s)-f_{*}(s)\right) d s \| \longrightarrow 0 \text { as } n \longrightarrow \infty .
\end{aligned}
$$

Thus, it follows by Lemma 2.6 that $\Theta \circ S_{F}$ is a closed graph operator. Further, we have $h_{n}(t) \in$ $\Theta\left(S_{F, x_{n}}\right)$. Since $x_{n} \rightarrow x_{*}$, therefore, Lemma 2.6 yields

$$
\begin{aligned}
h_{*}(t)= & \int_{0}^{t} \frac{(t-s)^{q-1}}{\Gamma(q)} f_{*}(s) d s-\frac{1}{2} \int_{0}^{T} \frac{(T-s)^{q-1}}{\Gamma(q)} f_{*}(s) d s \\
& +\frac{1}{4}(T-2 t) \int_{0}^{T} \frac{(T-s)^{q-2}}{\Gamma(q-1)} f_{*}(s) d s, \quad \text { for some } f_{*} \in S_{F, x_{*}} .
\end{aligned}
$$

Hence, we conclude that $\Omega$ is a compact multivalued map, u.s.c. with convex closed values. Thus, all the assumptions of Lemma 2.6 are satisfied and so by the conclusion of Lemma 2.6, $\Omega$ has a fixed point $x$ which is a solution of the problem (1.2).

Remark 3.2. If we take $F(t, y)=\{f(t, y)\}$, where $f:[0, T] \times \mathbb{R} \rightarrow \mathbb{R}$ is a continuous function, then our results correspond to a single-valued problem (a new result).

\section{Applications}

As an application of Theorem 3.1, we discuss two cases in relation to the nonlinearity $F$ in (1.2), namely, $F$ has (a) sublinear growth in its second variable (b) linear growth in its second variable (state variable). In case of sublinear growth, there exist functions $\eta(t), \rho(t) \in$ $L^{1}\left([0, T], \mathbb{R}_{+}\right), \mu \in[0,1)$ such that $\|F(t, x)\| \leq \eta(t)|x|^{\mu}+\rho(t)$ for each $(t, x) \in[0, T] \times \mathbb{R}$. 
In this case, $m_{r}(t)=\eta(t) r^{\mu}+\rho(t)$. For the linear growth, the nonlinearity $F$ satisfies the relation $\|F(t, x)\| \leq \eta(t)|x|+\rho(t)$ for each $(t, x) \in[0, T] \times \mathbb{R}$. In this case $m_{r}(t)=\eta(t) r+\rho(t)$ and the condition (3.1) modifies to $\|\eta\|_{L^{1}}<4 \Gamma(q) /(5+q) T^{q-1}$. In both the cases, the antiperiodic problem (1.2) has at least one solution on $[0, T]$.

\section{Examples}

(a) We consider $\|F(t, x)\| \leq \eta(t)|x|^{1 / 3}+\rho(t)$ and $T=1$ in (1.2). Here, $\eta(t), \rho(t) \in L^{1}\left([0,1], \mathbb{R}_{+}\right)$. Clearly $F(t, x)$ satisfies the assumptions of Theorem 3.1 with $0<4 \Gamma(q) /(5+q)$ (condition (3.1)). Thus, by the conclusion of Theorem 3.1, the antiperiodic problem (1.2) has at least one solution on $[0,1]$.

(b) As a second example, let $F(t, x)$ be such that $\|F(t, x)\| \leq\left(1 /(1+t)^{2}\right)|x|+e^{-t}$ and $T=1$ in (1.2). In this case, (3.1) takes the form $1 / 2<4 \Gamma(q) /(5+q)$. As all the assumptions of Theorem 3.1 are satisfied, the antiperiodic problem (1.2) has at least one solution on $[0,1]$.

\section{Acknowledgments}

The authors are grateful to the referees for their valuable suggestions that led to the improvement of the original manuscript. The research of V. Otero-Espinar has been partially supported by Ministerio de Educacion y Ciencia and FEDER, Project MTM2007-61724, and by Xunta de Galicia and FEDER, Project PGIDIT06PXIB207023PR.

\section{References}

[1] B. Ahmad and J. J. Nieto, "Existence results for nonlinear boundary value problems of fractional integrodifferential equations with integral boundary conditions," Boundary Value Problems, vol. 2009, Article ID 708576, 11 pages, 2009.

[2] B. Ahmad and S. Sivasundaram, "Existence and uniqueness results for nonlinear boundary value problems of fractional differential equations with separated boundary conditions," to appear in Dynamic Systems and Applications.

[3] B. Ahmad and J. J. Nieto, "Existence results for a coupled system of nonlinear functional differential equation with three-point boundary value problem," preprint.

[4] V. Daftardar-Gejji and S. Bhalekar, "Boundary value problems for multi-term fractional differential equations," Journal of Mathematical Analysis and Applications, vol. 345, no. 2, pp. 754-765, 2008.

[5] G. H. Erjaee and S. Momani, "Phase synchronization in fractional differential chaotic systems," Physics Letters A, vol. 372, no. 14, pp. 2350-2354, 2008.

[6] V. Gafiychuk, B. Datsko, and V. Meleshko, "Mathematical modeling of time fractional reactiondiffusion systems," Journal of Computational and Applied Mathematics, vol. 220, no. 1-2, pp. 215-225, 2008.

[7] R. W. Ibrahim and M. Darus, "Subordination and superordination for univalent solutions for fractional differential equations," Journal of Mathematical Analysis and Applications, vol. 345, no. 2, pp. 871-879, 2008.

[8] A. A. Kilbas, H. M. Srivastava, and J. J. Trujillo, Theory and Applications of Fractional Differential Equations, vol. 204 of North-Holland Mathematics Studies, Elsevier Science, Amsterdam, The Netherlands, 2006.

[9] S. Ladaci, J. J. Loiseau, and A. Charef, "Fractional order adaptive high-gain controllers for a class of linear systems," Communications in Nonlinear Science and Numerical Simulation, vol. 13, no. 4, pp. 707-714, 2008.

[10] M. P. Lazarević, "Finite time stability analysis of $\mathrm{PD}^{\alpha}$ fractional control of robotic time-delay systems," Mechanics Research Communications, vol. 33, no. 2, pp. 269-279, 2006. 
[11] I. Podlubny, Fractional Differential Equations, vol. 198 of Mathematics in Science and Engineering, Academic Press, San Diego, Calif, USA, 1999.

[12] S. Z. Rida, H. M. El-Sherbiny, and A. A. M. Arafa, "On the solution of the fractional nonlinear Schrödinger equation," Physics Letters A, vol. 372, no. 5, pp. 553-558, 2008.

[13] S. G. Samko, A. A. Kilbas, and O. I. Marichev, Fractional Integrals and Derivatives: Theory and Applications, Gordon and Breach, Yverdon, Switzerland, 1993.

[14] S. Zhang, "Existences of solutions for a boundary value problem of fractional order," Acta Mathematica Scientia, vol. 26, no. 2, pp. 220-228, 2006.

[15] B. Ahmad and J. J. Nieto, "Existence and approximation of solutions for a class of nonlinear impulsive functional differential equations with anti-periodic boundary conditions," Nonlinear Analysis: Theory, Methods \& Applications, vol. 69, no. 10, pp. 3291-3298, 2008.

[16] B. Ahmad and J. J. Nieto, “Existence of solutions for anti-periodic boundary value problems involving fractional differential equations via Leray-Schauder degree," preprint.

[17] Y. Chen, J. J. Nieto, and D. O'Regan, "Anti-periodic solutions for fully nonlinear first-order differential equations," Mathematical and Computer Modelling, vol. 46, no. 9-10, pp. 1183-1190, 2007.

[18] D. Franco, J. J. Nieto, and D. O’Regan, "Anti-periodic boundary value problem for nonlinear first order ordinary differential equations," Mathematical Inequalities $\mathcal{E}$ Applications, vol. 6, no. 3, pp. 477485, 2003.

[19] D. Franco, J. J. Nieto, and D. O'Regan, “Existence of solutions for first order ordinary differential equations with nonlinear boundary conditions," Applied Mathematics and Computation, vol. 153, no. 3, pp. 793-802, 2004.

[20] B. Liu, "An anti-periodic LaSalle oscillation theorem for a class of functional differential equations," Journal of Computational and Applied Mathematics, vol. 223, no. 2, pp. 1081-1086, 2009.

[21] Z. Luo, J. Shen, and J. J. Nieto, "Antiperiodic boundary value problem for first-order impulsive ordinary differential equations," Computers $\mathcal{E}$ Mathematics with Applications, vol. 49, no. 2-3, pp. 253261, 2005.

[22] K. Wang and Y. Li, "A note on existence of (anti-)periodic and heteroclinic solutions for a class of second-order odes," Nonlinear Analysis: Theory, Methods E Applications, vol. 70, no. 4, pp. 1711-1724, 2009.

[23] S. Abbasbandy, J. J. Nieto, and M. Alavi, “Tuning of reachable set in one dimensional fuzzy differential inclusions," Chaos, Solitons E Fractals, vol. 26, no. 5, pp. 1337-1341, 2005.

[24] Y.-K. Chang, W.-T. Li, and J. J. Nieto, "Controllability of evolution differential inclusions in Banach spaces," Nonlinear Analysis: Theory, Methods E Applications, vol. 67, no. 2, pp. 623-632, 2007.

[25] M. Frigon, "Systems of first order differential inclusions with maximal monotone terms," Nonlinear Analysis: Theory, Methods \& Applications, vol. 66, no. 9, pp. 2064-2077, 2007.

[26] J. J. Nieto and R. Rodríguez-López, "Euler polygonal method for metric dynamical systems," Information Sciences, vol. 177, no. 20, pp. 4256-4270, 2007.

[27] G. V. Smirnov, Introduction to the Theory of Differential Inclusions, vol. 41 of Graduate Studies in Mathematics, American Mathematical Society, Providence, RI, USA, 2002.

[28] Y.-K. Chang and J. J. Nieto, “Existence of solutions for impulsive neutral integrodi-differential inclusions with nonlocal initial conditions via fractional operators," Numerical Functional Analysis and Optimization, vol. 30, no. 3-4, pp. 227-244, 2009.

[29] Y.-K. Chang, J. J. Nieto, and W.-S. Li, “On impulsive hyperbolic differential inclusions with nonlocal initial conditions," Journal of Optimization Theory and Applications, vol. 140, no. 3, pp. 431-442, 2009.

[30] J. Henderson and A. Ouahab, "Fractional functional differential inclusions with finite delay," Nonlinear Analysis: Theory, Methods \& Applications, vol. 70, no. 5, pp. 2091-2105, 2009.

[31] W.-S. Li, Y.-K. Chang, and J. J. Nieto, "Existence results for impulsive neutral evolution differential inclusions with state-dependent delay," Mathematical and Computer Modelling, vol. 49, no. 9-10, pp. 1920-1927, 2009.

[32] A. Ouahab, "Some results for fractional boundary value problem of differential inclusions," Nonlinear Analysis: Theory, Methods \& Applications, vol. 69, no. 11, pp. 3877-3896, 2008.

[33] Y.-K. Chang and J. J. Nieto, "Some new existence results for fractional differential inclusions with boundary conditions," Mathematical and Computer Modelling, vol. 49, no. 3-4, pp. 605-609, 2009.

[34] K. Deimling, Multivalued Differential Equations, vol. 1 of de Gruyter Series in Nonlinear Analysis and Applications, Walter de Gruyter, Berlin, Germany, 1992. 
[35] S. Hu and N. Papageorgiou, Handbook of Multivalued Analysis, Theory Mathematics and Its Applications, Kluwer Academic Publishers, Dordrecht, The Netherlands, 1997.

[36] H. F. Bohnenblust and S. Karlin, "On a theorem of Ville," in Contributions to the Theory of Games. Vol. I, Annals of Mathematics Studies, no. 24, pp. 155-160, Princeton University Press, Princeton, NJ, USA, 1950.

[37] A. Lasota and Z. Opial, "An application of the Kakutani-Ky Fan theorem in the theory of ordinary differential equations," Bulletin de l'Académie Polonaise des Sciences. Série des Sciences Mathématiques, Astronomiques et Physiques, vol. 13, pp. 781-786, 1965. 